\section{Drehmaschinen auslegen}

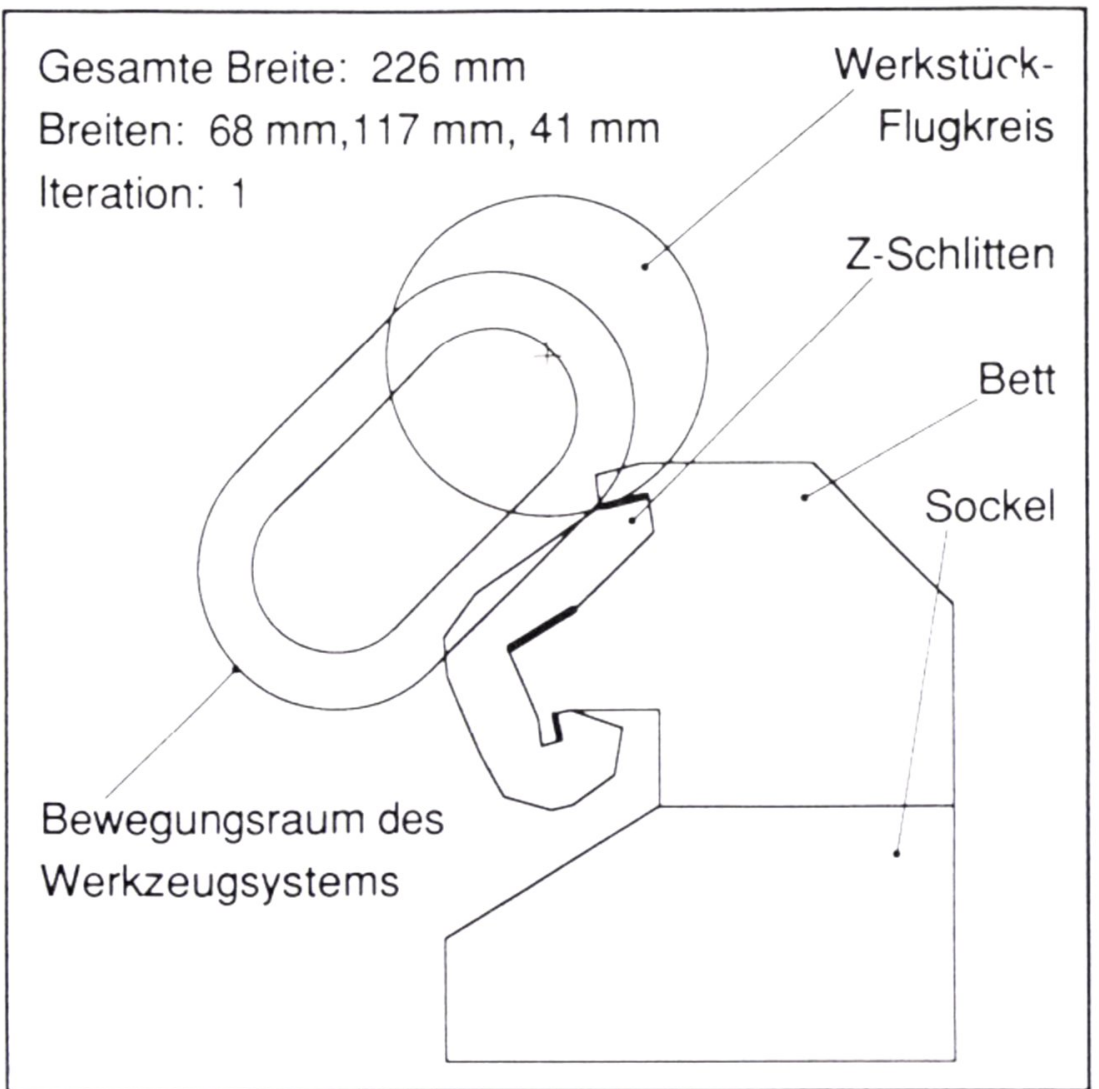

Zweck der rechnerunterstützten Optimierung von Drehmaschinenkonzepten ist vor allem die Erzielung einer leichteren und kostengünstigeren Gestellkonstruktion. Berechnung an ausgeführten CNC-Drehmaschinen deuten darauf hin, daß mit dem hier vorgestellten Verfahren nennenswerte Einsparungen möglich sind. S. 239

\section{Entwicklungen mit Features}

Im Rahmen eines Esprit-Projekts wurde ein Featuremodelliersystem als Bestandteil eines Konstruktions- und Arbeitsplanungssystems entwickelt. In diesem Beitrag wird beschrieben, wie die integrierte Verarbeitung geometrischer, technologischer und funktiona ler Information in der Praxis vonstatten geht

S. 247

\section{Produktorientiertes Modellieren}

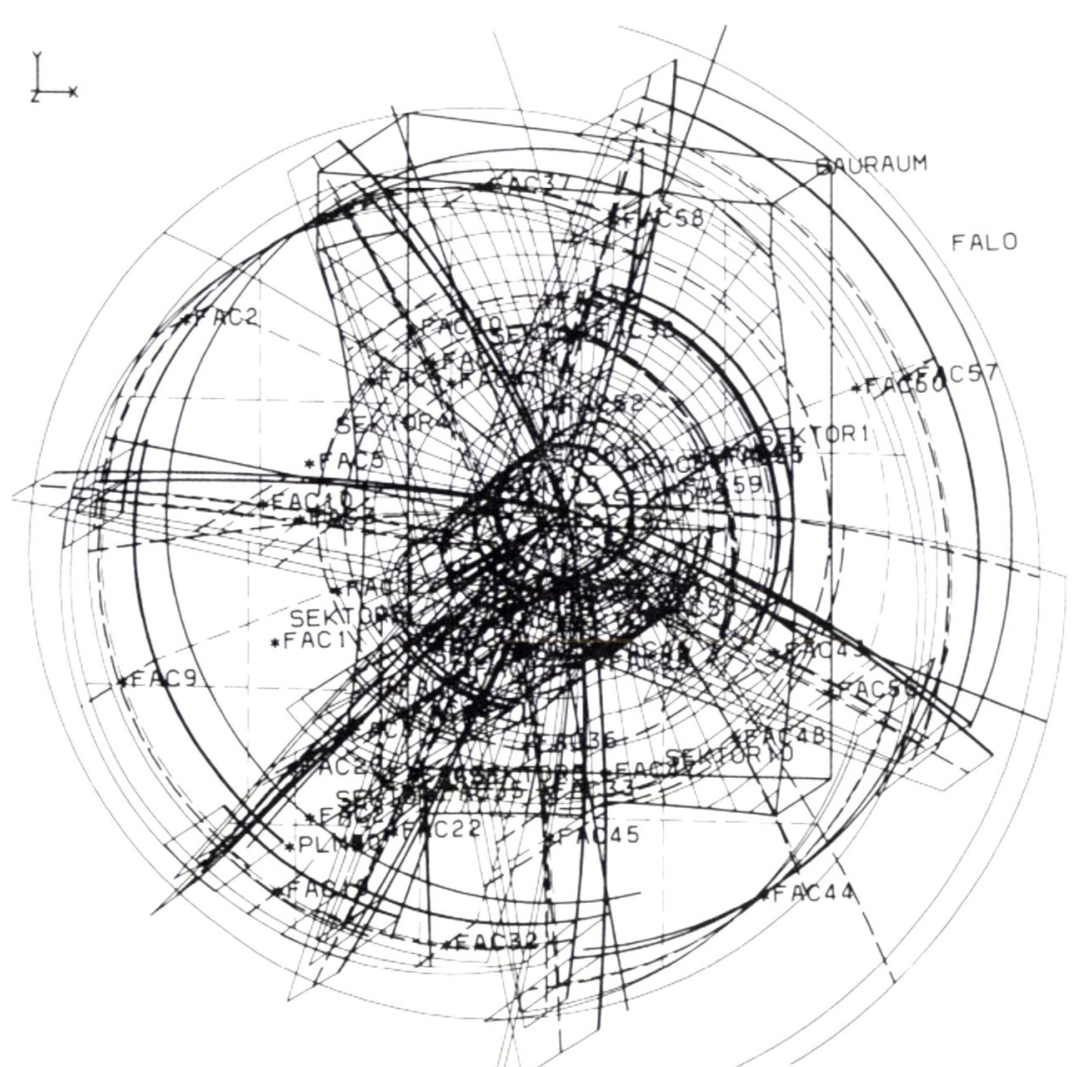

Das Wissen um die Konstruktionsabsicht hebt den Dialog zwischen System und Konstrukteur auf eine neue Stufe. Das Modelliersystem erhält eine gewisse "Intelligenz" und eine anwendungsspezifische „Verhaltensweise". In diesem Fall wurde ein weitgehender Ansatz für die produkt orientierte Modellierung von Scheinwerfern und Leuchten realisiert.

\section{Editorial}

Anwendungsorientierte CAD-Systeme.

Notizen

aus Branchen und Unternehmen ..................... 236

zur Person. ................................ 237

zu Veranstaltungen ......................... 238

Rechnerunterstützte Konstruktion und Planung

G. Spur, A. Schüle: Optimierung von Drehmaschinenkonzepten durch Statikanalyse

W. F. Lastowski: Strukturanalyse für Konstrukteure. . . . . . . . . . . 243 Normteile in CAD-Zeichnungen integrieren . . . . . . . . . . . . . . . 246

F.-L. Krause, S. Kramer, E. Rieger: Featurebasierte Produktentwicklung

Neuer Farb-Tintenstrahlplotter

Schnelles Erzeugen von zweidimensionalen Darstellungen

C. Vogt, J. Reissner: Kommunikations-Interface integriert Expertensystem-Shell und CAD-System

Normteile und Sachmerkmalleisten unter Windows. . . . . . . . . . . . 256

Rechnergestützt Protoypen und Versuche reduzieren . . . . . . . . . 256

B. Wördenweber: Produktorientiertes Modellieren. . . . . . . . . . . 257

Temperaturen in Bauteilen bestimmen . . . . . . . . . . . . . . 260

CAD-System für die Elektrotechnik . . . . . . . . . . . . . . . . 260

M. Geiger, M. Hoffmann, B. Kluge: Inferenzmaschine für ein Biegestadienplanungssystem ..................... 261

J. Xu: Rechnerunterstützte Arbeitsplanung . . . . . . . . . . . . . 265

Rechneranwendung in Fertigung und Montage

A. Potthast, N. Zughaibi, I. Suwalski: Modellgestützte Qualitätssicherung an CNC-Werkzeugmaschinen ............... 269

Objektorientierte Software mit neuen Merkmalen

C. Lochner, D. Widmaier: Objektorientiertes Fertigungsleitstandskonzept

Unix-Datenbank mit neuen Entwicklungswerkzeugen . . . . . . . . 278

Verbindung heterogener Hardware-Welten

CIM - Rechnerintegrierter Fachbetrieb

H. F. Binner: Durchgängige Regelkreise sichern die Termineinhaltung bei Einzelfertigern.

Unkoordinierter Netzwerk-Betrieb gefährdet die Wirtschaftlichkeit von Netzen

Netzwerk-Betriebssysteme anpassen

K.-G. Schade: Wege zum wirtschaftlichen Einsatz von technischen Informationssystemen

Mechanische Teile mit Leuchtdioden realistisch darstellen . . . . . . 286

Engpaß bei der Implementierung offener Systeme

286

\section{Report}

CAD $\cdot$ CAM $\cdot$ CIM

Fabrikanlagen und Materialfluß 
Sonderteil: CAD/CAM/CIM

CA $77-$ CA 152

\section{CAD-Nachrichten der GI}

\section{Notizen}

aus der Branche

CA 82

Leitartikel

G. Spur: Objektorientierte Systeme für anwenderfreundliche Applikationen

Maschinen- und Anlagenbau

G. Meyer: Angebote und Aufträge rechnerunterstützt bearbeiten

CA 86

H. F. W. Schramm: CAD als Wegbereiter der Wettbewerbsfähigkeit

CA 91

Werkzeug- und Formenbau

B. Argetsinger: NC-Programmiersystem unterstützt Fräsbearbeitung von Spritzgießwerkzeugen

CA 96

A. Christinger, T. Weninger: Spritzgießwerkzeuge mit MCAE-

Software entwickeln.

H. Bartnik, W. Taschner: Rechnereinsatz für ein angenehmes Bad

F. Tschacha: Durch CAD/CAM vom Handwerk zur Industriefertigung

CA 110

Rechnerunterstützte Berechnungsverfahren

M. Dutly: Trauen Sie den Meßergebnissen oder der Finite Elemente-Methode?

CA 114

Zulieferindustrie

A. Gührer, T. Rückle: Technische Informationen einheitlich verwalten

CA 120

Kunststoffverarbeitung

U. Steinmeyer, R. Metzler, G. König: Kunststofftechnik investiert

CA 128

Qualitätssicherung

S. Sprang, S. Steck: CAD-integrierte Off-Line-Programmierung von Koordinatenmeßgeräten.

CA 137

Kurzberichte

Neue Software-Generation für die CAD/CAM-Umgebung

CA 85

Plotten in LAN- und Mehrplatz-Umgebungen

NC-Programmiersystem für Workstation-Rechner

CA 94

CA 106

3-D-Komponentenbibliothek / Mehrkörpersimulation

CA 108

Report

CA 147

Supplement: Laser-Praxis

LS 1-LS 76

R. Wollermann-Windgasse: Die derzeitigen Grenzen der Lasertechnologie überwinden

C. Olainek: Laser und Roboter als Team

LS 25

Vollständiges Inhaltsverzeichnis

\section{Fehler an CNC-Maschinen} diagnostizieren

Das hier geschilderte Verfahren zur Fehlerdiagnose und Maschinenend abnahme ist kostengünstig und wenig fehleranfällig, da es ohne zusätzliche Sensorik auskommt. Es basiert auf einer modellgestützten Diagnose, bei der die Merkmale für den Zustand des zu überwachenden Objekts aus den gemessenen Signalverläufen über geeignete Schätzmethoden gewonnen werden. Das in die CNC integrierbare Diagnosesystem wurde als Modul zum Nachrüsten vorhandener Werkzeugmaschinensteuerungen entwickelt.

\section{Sonderteil: $C A D / C A M / C I M$}

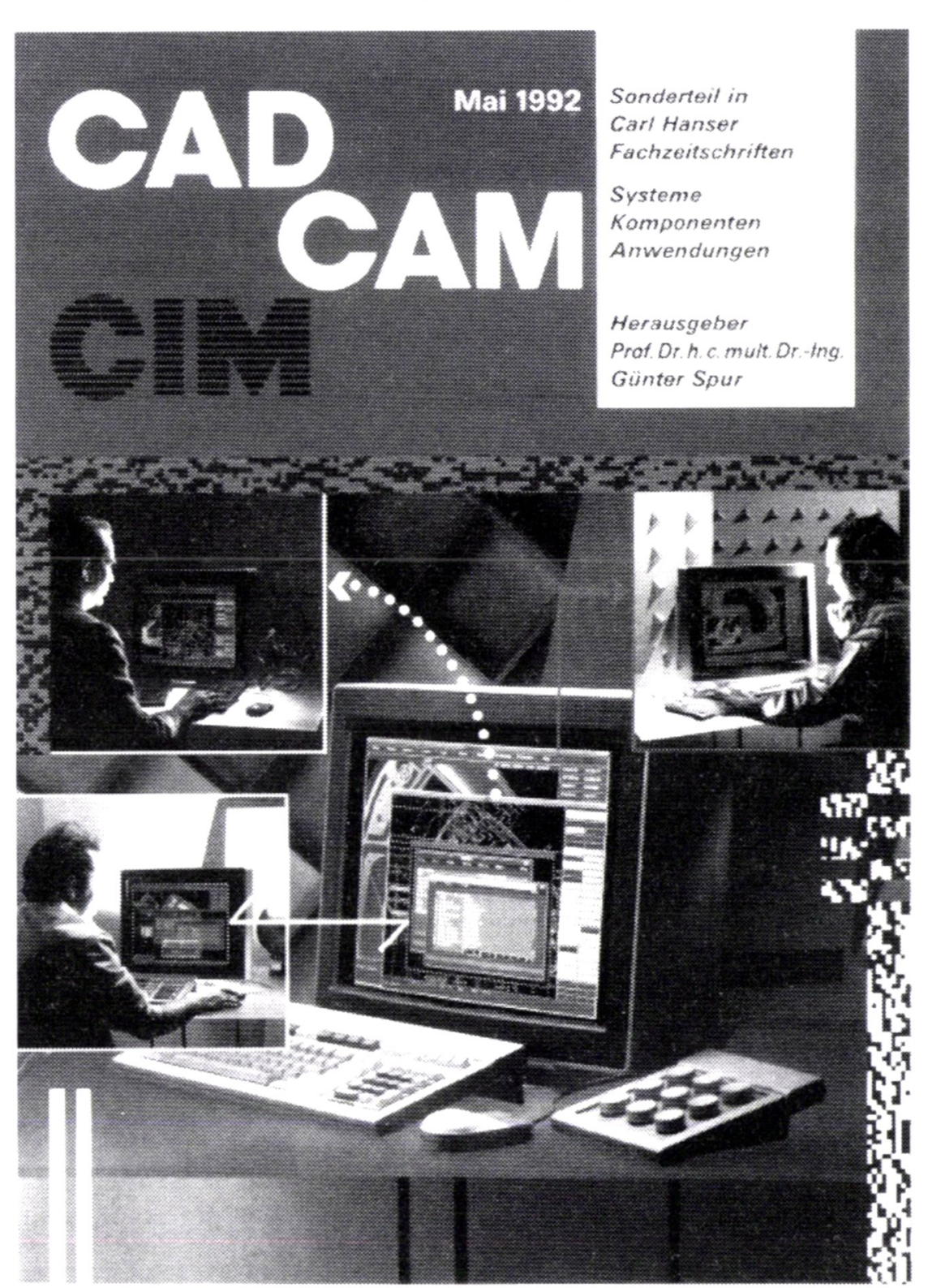

Supplement: Laser-Praxis

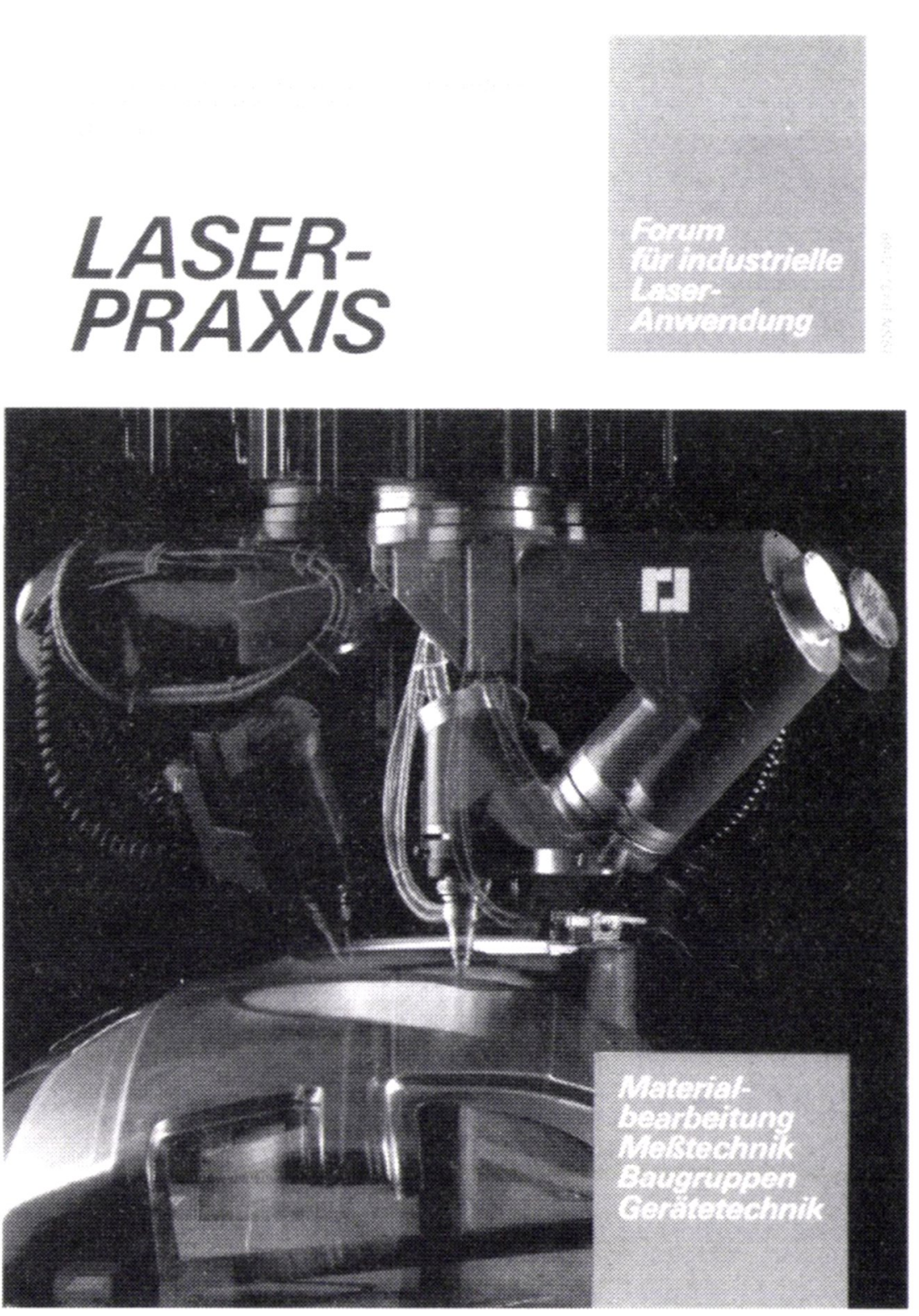

